

\title{
医学研究与发表系列讲坛之 “万用模版”研习班精彩回顾
}

\section{陈甫州教授简介}

美国奥克拉荷马大学化学暨生化系博士, 现任职于台湾台中荣民总医院医学研究部研究 员兼任干细胞中心主任; 静宜大学应用化学系 合聘教授

曾获得中华民国医检学会钟慧芬纪念奖, 行政院退辅会特优论文奖, 千禧年脑中风优秀 论文奖, 严庆玲基础医学杰出研究奖, 国科会 研究等多项荣誉。

近年来, 陈教授倾全力教导学术界教授、 医师、博士生、硕士生的英文论文写作及技巧, 以独创的「万用模版英文论文写作法」为基础, 从四段式简介、实验、结果与讨论之撰写方式 为主轴, 使用模版化空白表格, 逐步填入论文 之本体结构, 并应用现成之免费网路软体与电 脑语句库, 协助科技人员熟稔英文科技论文的 表达方法, 让英文期刊的读者容易接受其所欲 表达之意念, 并在不同模版撰写过程中, 提升 其英文语意的表达与写作技巧, 这种新的英文 写作方式能带给研究学者更便利的研究辅助工 具, 同时也透过一对一的写作工作坊辅导, 帮 助年轻的研究学者发表论文。陈教授是一个虔 诚的基督徒, 除了在教会中教导, 也热心参与 社会工作, 目前更积极地协助家庭婚姻之辅导 工作, 也参与学生就学辅导, 更关心社会青年 的恋爱与就业辅导, 在他人生下半场的规划中, 披带着耶稣基督的虔诚信仰, 将人们的需要转 变成他的社会责任, “生活信仰化, 信仰生活化” 是他一生事奉的圭臬。
美捷登精心准备每一场讲 座，从前期会务到后期服务，每 一个环节都力争让每一位学员感 到舒心、安心。2016 年 3 月, 短短的半个月时间，美捷登走过 了 4 个城市，举办了 6 场 “万用 模版”研习班。每一场讲座现场 都是座无虚席; 每一场讲座结束 都是好评如潮。不论是 “万用模 版”独创者陈教授，还是应邀而 来的嘉宾，都为听众带来了简单 易懂而又超级实用的技巧和经 验, 让听众在轻松、愉快的学习 环境中体验前所未有的头脑风 暴, 相信每一位听众都觉得不虚 此行。

陈教授独创的 “万用模版” 英文论文写作法是本系列研习班 的重点和亮点, 他创新的写作思 维让每一位学员满载而归, 深人 浅出的授课方式, 轻松诙谐的课 堂氛围, 更是让每一位学员都沉 浸其中，意犹未尽。

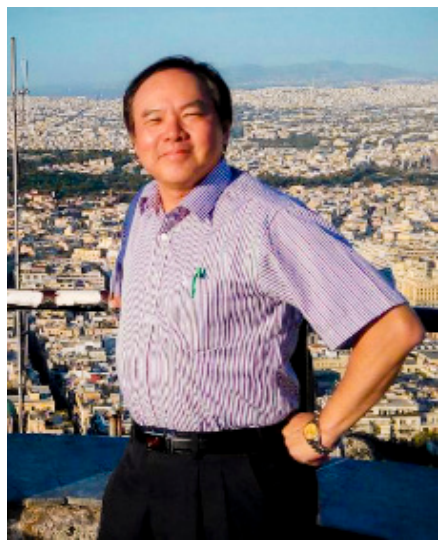

内容简介

英文论文写作的目的在于 传达科学研究者的研究理念和成 果，如果作者以清晰、流畅的语 句, 按照期刊的四段式结构: 简 介、实验、结果与讨论逻辑的段 落撰写, 能使读者简易阅读, 清 楚理解其内容。

通过定义英文论文的结构格 式为特定的模版，按 “模版” 逐 步填人论文本体, 化繁为简, 并 通过电脑语句库相关软件辅助 英文写作, 可以准确地表达作者 之研究意念与内涵, 使读者容易 接受其理念。本系列课程将对此 结构化技巧进行系统的介绍和辅 导，预期这种新的 “万用模版” 英文论文写作法将提供研究学者 们更轻松、更便利的写作辅助工 具, 写出流利的英文论文, 发表 高水平、高质量的科研论文。 


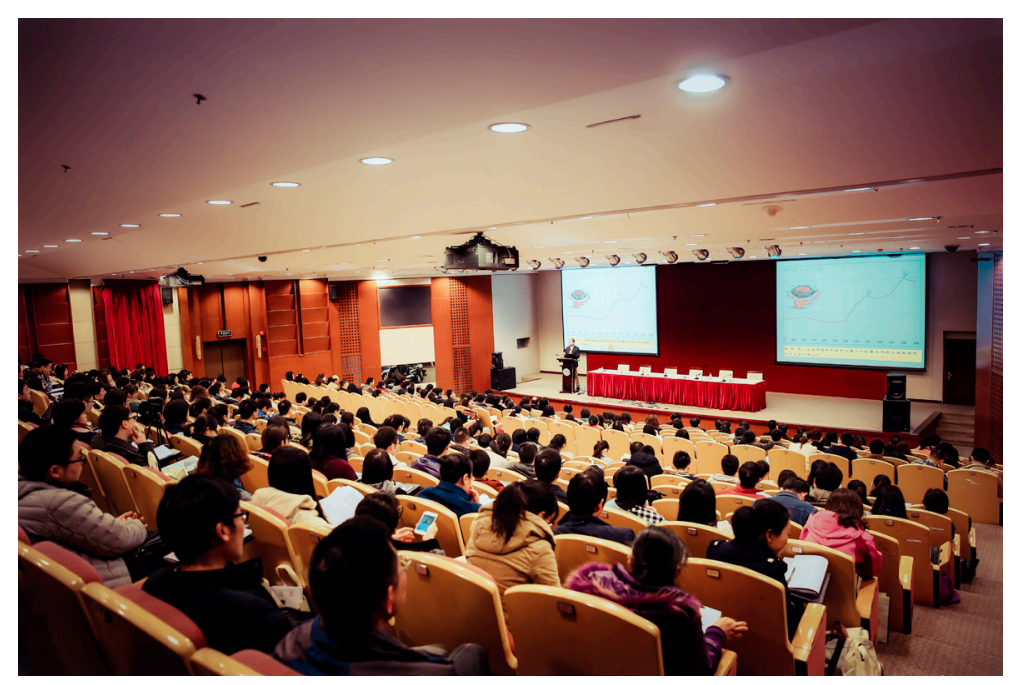

陈甫州教授的讲课吸引了莘莘学子, 座无虚席一一上海金山站

\section{创作历程}

在研究许多中、英文教导 英文论文写作书籍后，并经过几 年的研究和思索, 为了让年轻学 者能够掌握一套易懂、易学、又 具备实战的英文写作技能，陈 教授针对华人英文论文写作的困 境与迷失，透过模版化的文章 结构概念，利用分解结合的简 易写作逻辑，编写了一套 “万用 模版”英文论文写作法。通过分 解论文简介 (introduction)、方法 (method)、结果 (results)、讨论 和结论 (discussion \& conclusion) 的基本架构与外形（如右图）, 再按照段落中每一个句子应有的 语义结构, 并检视句与句之间彼 此的起、承、转、合的结构连结 关系, 最后将每一个句子按类似 的结构, 在各段落中的语文属性 与排列, 定义出每一篇文章各个 段落与各分段的定型基本结构,

\section{“魔力” 精髓}

孰悉使用 “万用模版” 就能 将论文结构先套好招, 通过论文 的结构学, 明确在第一段写什 么? 第二段写什么? 然后, 回到 第一段的第一句要写什么? 第二 句如何衔接? 结论怎么下? 随 后，有关英文论文的撰写，还可 以通过电脑语句库相关软件的辅 助, 从而令人担心的 “破英文” 就自然迎刃而解了。如果有不会 写的段落或句子, 可以先跳过或 做一个记号或先用中文表达, 通 过「万用模版」撰写论文的时候, 只要一有时间就可以写一小段, 随时可以停, 任何时候回到模版 时, 都可以清楚地知道整篇论文 中还缺那一段落或那一句, 不容 易漏掉一些重要的关键内容, 让 论文结构与模版都能够清楚地呈 现，这是应用 “万用模版” 英文 论文写作法的精髓。

欢迎各单位提前预订课

绘制出了一张实用的 “万用模 版”, 至于这些模版语文结构的 定义是什么? 又该如何进行运用 呢? 陈甫州教授将通过一系列课 程详细阐述, 听者必将恍然大悟、 如获至宝!
程, 如有意向, 请联系讲

座 / 培训接洽人: 秦雪文 15094028338 